\title{
Mutations in ORP1 Conferring Oxathiapiprolin Resistance Confirmed by Genome Editing using CRISPR/Cas9 in Phytophthora capsici and P. sojae
}

\author{
Jianqiang Miao, Yuandong Chi, Dong Lin, Brett M. Tyler, ${ }^{\dagger}$ and Xili Liu ${ }^{\dagger}$
}

First and fifth authors: State Key Laboratory of Crop Stress Biology for Arid Areas, College of Plant Protection, Northwest A\&F University, Yangling 712100, China; first, second, third, and fifth authors: Department of Plant Pathology, College of Plant Protection, China Agricultural University, Beijing 100193, China; and first and fourth authors: Center for Genome Research and Biocomputing and Department of Botany and Plant Pathology, Oregon State University, Corvallis 97331.

Accepted for publication 4 July 2018.

\begin{abstract}
Oxathiapiprolin is a novel fungicide that was recently registered in a number of countries to control plant-pathogenic oomycetes such as Phytophthora capsici. In our previous study, point mutations G770V and G839W in oxysterol binding protein-related protein 1 (ORP1) were detected in oxathiapiprolin-resistant $P$. capsici isolates (PcORP1). Here, we used the CRISPR/Cas9 system to verify the effects of these two point mutations on $P$. capsici phenotypes. Transformants containing heterozygous G770V and G839W mutations in PcORP1 showed high levels of oxathiapiprolin resistance. The G770V transformants showed otherwise similar phenotypes compared with the wild-type isolate BYA5, including sporangia and zoospore production, cyst germination, and pathogenicity.

However, two independent transformants with heterozygous G839W mutations in PcORP1 could not produce sporangia. Three transformants with an unexpected point mutation in PcORP1 ( $\triangle$ N837) showed high oxathiapiprolin resistance, and either similar or significantly reduced fitness compared with BYA5. The same deletion $(\Delta \mathrm{N} 837)$ was confirmed to confer oxathiapiprolin resistance in P. sojae by using CRISPR/Cas9. These homozygous $P$. sojae mutants also showed either similar or strongly reduced fitness compared with the wild-type parent isolate P6497. These results improve our understanding of oxathiapiprolin resistance in Phytophthora spp., and will be useful for the development of novel oxysterol-binding protein homolog inhibitor fungicides.
\end{abstract}

Oomycetes are among the most problematic group of diseasecausing organisms in agriculture. Well-known oomycete plant pathogens include Phytophthora spp., Pythium spp., and downy mildew agents (Blair et al. 2008; Derevnina et al. 2016; Fichtner et al. 2012; Meitz-Hopkins et al. 2014).

Generally, chemical fungicides still play an important role in the management of diseases caused by oomycetes. However, most oomycetes exhibit a tremendous ability to adapt to chemicals through the emergence of genetically resistant strains (Gisi and Sierotzki 2008; Urban and Lebeda 2006; Ziogas et al. 2006).

Oxathiapiprolin, developed by DuPont, belongs to a novel fungicide class: namely, oxysterol-binding protein homolog inhibitors (FRAC 2017; Pasteris et al. 2016). It has excellent preventive, curative, and remanence efficacy against many important plant oomycete diseases, such as Phytophthora blights and downy mildews (Bittner and Mila 2017; Cohen 2015; Ji and Csinos 2015; Ji et al. 2014). It can be applied through foliar sprays (ground, aerial, and chemigation), soil applications (in-furrow and banded), or soil drenches. Application rates are much lower than regular fungicides, ranging from 6.42 to $61.78 \mathrm{lb}$. of active ingredient per square kilometer. Currently, oxathiapiprolin has been registered in China, the United States, the European Union, New Zealand, Australia, and some other countries to control plant-pathogenic oomycetes.

Because of its single-site mode of action, oxathiapiprolin readily selected resistant mutants in $P$. capsici and $P$. nicotianae in the

${ }^{\dagger}$ Corresponding authors: Brett M. Tyler; E-mail: brett.tyler@oregonstate.edu; and Xili Liu; E-mail: seedlingxili@163.com

Funding: This work was funded by the National Natural Science Foundation of China (numbers 31471791 and 31730075 ).

(C) 2018 The American Phytopathological Society laboratory (Andreassi et al. 2013; Bittner et al. 2017; Miao et al. 2016). In oxathiapiprolin-resistant mutants, nine point mutations have been detected in the oxysterol-binding domain of the target protein, oxysterol binding protein-related protein 1 (ORP1). According to the proposal of Mair et al. (2016) to unify the labeling of amino acids in fungicide target proteins, the oxathiapiprolin target protein in $P$. infestans was used as the archetype protein and numerical labels on the position of the residue in it were assigned. In P. capsici ultraviolet mutagenesis mutants, L733W, S768I/F/K/Y, G770A/I/P/V/L, N837I/F/Y, G839W, P861H, L863W/F, and I877F/ $\mathrm{Y}$ were detected in $P$. capsici ORP1 (PcORP1) (Andreassi et al. 2013). Andreassi et al. (2013) obtained grape downy mildew isolates from field trials where oxathiapiprolin had been continuously used for 4 years, and detected two amino acid changes (N837I and L863W) in resistant isolates. Bittner et al. (2017) also detected G770V and $\Delta \mathrm{G} 818 / \mathrm{F} 819$ mutations in $P$. nicotianaeresistant mutants produced by UV radiation. G770V and G839W were also found in $P$. capsici fungicide adaptation mutants, and G839W was confirmed by traditional overexpression transformation to confer oxathiapiprolin resistance (Miao et al. 2016). Because the mutants containing G770V showed significantly reduced fitness, we did not verify whether this point mutation confers resistance to oxathiapiprolin.

Distinct from the zinc-finger nucleases and transcription activator-like effector nucleases, the RNA-guided Cas9 endonucleases from the microbial clustered regularly interspaced short palindromic repeats (CRISPR) have emerged as a powerful tool for mediating genome alterations with high precision (Hsu et al. 2014). Recently, the CRISPR/Cas9 system was established in $P$. sojae as an effective gene-editing method in oomycetes (Fang and Tyler 2016; Fang et al. 2017). Here, we used CRISPR/Cas9 to confirm that the point mutations G770V and G839W, as well as a novel mutation, $\Delta \mathrm{N} 837$, confer resistance to oxathiapiprolin in $P$. capsici, and to determine how they affect the biological characteristics of $P$. capsici and $P$. sojae. 


\section{MATERIALS AND METHODS}

Fungicide. Technical-grade oxathiapiprolin (96.7\% active ingredient) was kindly provided by Dupont Crop Protection. It was accurately weighed and dissolved in dimethyl sulfoxide (DMSO) to prepare a stock solution at $10^{4} \mu \mathrm{g} / \mathrm{ml}$, which was stored in darkness at $4^{\circ} \mathrm{C}$ until required.

Phytophthora strains and growth conditions. The wildtype $P$. capsici strain BYA5, the reference $P$. sojae isolate P6497 (race 2), and all transgenic isolates used in this study were routinely grown on $10 \% \mathrm{~V} 8$ medium at $25^{\circ} \mathrm{C}$ in darkness. The sensitivity of wild-type isolates and all transgenic lines to oxathiapiprolin was determined using the mycelia growth assay described by Pang et al. (2013). The final concentration of DMSO in the medium was standardized at $0.1 \%(\mathrm{vol} / \mathrm{vol})$. Each treatment consisted of three replicates and all experiments were repeated twice.

Plasmid construction. The sequences of the PCORPI and $P$. sojae ORP1 (PsORPl) genes were obtained by searching the $P$. capsici genome, v11.0 (https://genome.jgi.doe.gov/Phyca11/Phyca11. home.html), and the P. sojae genome, v3.0 (https://genome.jgi.doe. gov/Physo3/Physo3.home.html). The full-length genes of PCORP1 from $P$. capsici BYA5 and PsORP1 from P. sojae P6497 were amplified using CloneAmp HiFi PCR Premix (Takara) and sequenced. All other fragments were also amplified using CloneAmp HiFi PCR Premix (Takara).

According to our previous study (Miao et al. 2016), point mutations at nucleotides $2,173(\mathrm{G} \rightarrow \mathrm{T})$ and $2,379(\mathrm{G} \rightarrow \mathrm{T})$ in PcORP1 produced the amino acid substitutions G770V and G839W, respectively, in oxathiapiprolin-resistant mutants of $P$. capsici. To use the CRISPR/Cas9 system to confirm whether these point mutations confer resistance to oxathiapiprolin, constructs encoding two singleguide RNA (sgRNA) (sg2180-GAAACCATTCAACCCGATCC and sg2358-GCTATGCTCAACACCAACAA) were cloned separately into plasmid PYF515 (Fang et al. 2017). The sgRNA were designed using the web tool sgRNA Designer (https://portals.broadinstitute. org/gpp/public/) (Doench et al. 2014). Then, the sgRNA-encoding fragments were inserted into the all-in-one plasmid pYF515, according to the protocol of Fang et al. (2017). Flanking sequences, $1,000 \mathrm{bp}$ upstream and downstream of the mutation site, were amplified and infused into pBS-SK+ using the primers listed in Table 1 and the In-fusion HD Cloning Kit (Clontech), to create the basic donor plasmid.

Transformation of $\boldsymbol{P}$. capsici and $\boldsymbol{P}$. sojae. Polyethylene glycol-mediated protoplast transformations were conducted using previously described methods (Fang and Tyler 2016), with a minor modification. The wild-type $P$. sojae or $P$. capsici strain was cultured on a V8 agar plate. After 2 to 5 days, five to eight mycelium discs, cut from the edge of actively growing colonies, were grown into $50 \mathrm{ml}$ of nutrient pea broth to produce fresh hyphae for protoplast preparation. All other steps were performed as in the previous protocol.

To avoid the sgRNA-mediated Cas9 cleavage of the homologydirected repair (HDR) donor, the sgRNA recognition site was also mutated. In order to prevent the sgRNA-guided Cas9 from cutting the donor plasmid, the sgRNA binding residues in the donor plasmid were mutated without changing the encoded amino acids, producing the two donor plasmids pBS-HDR1 and pBS-HDR2, respectively (Fig. 1). PYF515-sg2358 was cotransformed together with pBS-HDR1 and pYF515-sg2180 was cotransformed with pBS-HDR2.

Transformants were transferred to V8 liquid medium containing G418 at $50 \mu \mathrm{g} / \mathrm{ml}$ or oxathiapiprolin at $0.005 \mu \mathrm{g} / \mathrm{ml}$ and incubated for 2 to 3 days at $25^{\circ} \mathrm{C}$ prior to analysis. The hyphae of the transformants were then collected for genomic DNA (gDNA) extraction. For verification of the transformants, the amplification products of the full-length $P c O R P 1$ or $P S O R P 1$ genes in all transformants were sequenced.

Nucleic acid extraction. Total gDNA was extracted according to Edwards et al. (1991), with some modifications. The mycelium sample was ground after freezing in liquid nitrogen. Then, $400 \mu \mathrm{l}$ of extraction buffer (200 mM Tris $\mathrm{HCl}$ [pH 7.5], $250 \mathrm{mM} \mathrm{NaCl}, 25 \mathrm{mM}$ EDTA, and $0.5 \%$ sodium dodecyl sulfate) was added, and the mix was vortexed for $10 \mathrm{~s}$ and centrifuged at 13,000 rpm for $10 \mathrm{~min}$, before $300 \mu \mathrm{l}$ of the supernatant was transferred to a new $1.5-\mathrm{ml}$ tube. Then, $300 \mu \mathrm{l}$ of isopropanol was added into the supernatant and chilled at $-20^{\circ} \mathrm{C}$ for $1 \mathrm{~h}$. Following centrifugation (13,000 rpm for $5 \mathrm{~min}$ ), the supernatant was removed and the pellet was vacuum dried. Then, the gDNA was suspended in $50 \mu \mathrm{l}$ of $\mathrm{H}_{2} \mathrm{O}$. For RNA extraction, mycelium was harvested from P. capsici or P. sojae isolates grown on V8 liquid medium for 2 or 3 days, respectively, and frozen at $-80^{\circ} \mathrm{C}$ until required. Total RNA was extracted from the frozen samples using the SV Total RNA Isolation kit (Promega Corp.), and cDNA was synthesized using the PrimeScript II First-Strand cDNA Synthesis Kit (Takara) according to the protocol of the manufacturers.

Phenotype analysis of $\boldsymbol{P}$. sojae and $\boldsymbol{P}$. capsica. Mycelial growth was determined after depositing a mycelial plug $(5 \mathrm{~mm}$ in diameter) on $10 \% \mathrm{~V} 8$ agar medium and incubating the plates for 7 days ( $P$. sojae) or 5 days ( $P$. capsici) in the dark at $25^{\circ} \mathrm{C}$.

To analyze sporangia production of $P$. sojae, 10 hyphal tip plugs ( $5 \mathrm{~mm}$ in diameter) cut from the edge of an actively growing culture of each strain were deposited in a plastic plate containing $20 \mathrm{ml}$ of

TABLE 1. Primers used in the study

\begin{tabular}{lll}
\hline Primer & \multicolumn{1}{c}{ Sequence $\left(5^{\prime}-3^{\prime}\right)$} & \multicolumn{1}{c}{ Application $^{z}$} \\
\hline P1 & GCTTGATATCGAATTCAAGTGGAAGCTTTGCCCAG & Amplification of upstream and downstream regions around codon 770 of $P c O R P 1$ \\
P2 & TAGAACTAGTGGATCCTCCGACTTCAACCACGAGATG & $\ldots$ \\
P3 & GCTTGATATCGATTCTCTTCGTCATGTGCGTGAGTCT & Amplification of upstream and downstream regions around codon 839 of $P c O R P 1$ \\
P4 & TAGAACTAGTGGATCCCAGGCTGAAGGTCGATGATGTAC & $\ldots$ \\
P5 & GCGATGCTGAATACGAATAAAGGGGCCTGTGCGTGTTAC & Synonymous mutation of sg2358 target sequence in pBS-HDR1 \\
P6 & TTTATTCGTATTCAGCATCGCGTTGGACTTGACGCTCAT & $\ldots$ \\
P7 & GAAGCCGTTTAATCCCATTCTCTGGGCGAAACATACCAGT & Synonymous mutation of sg2180 target sequence in pBS-HDR2 \\
P8 & GAGAATGGGATTAAACGGCTTCAGCTGTCCGATACTGTGG & $\ldots$ \\
P9 & GCTTGATATCGAATTCGGTGCTGCTGCGTCACGT & Amplification of upstream and downstream regions around codon 837 of $P$ soRP1 \\
P10 & TAGAACTAGTGGATCCGATTTACAAATCGATCGATCTAAAT & $\ldots$ \\
P11 & GCCATGCTGAATACGAAAGGTCCTGTGCGCGTGACGTT & Synonymous mutation of sg2381 target sequence in pBS-HDR3 \\
P12 & CCTTTCGTATTCAGCATGGCGTTGGACTTGACGCTCAT & $\ldots$ \\
P13 & CACCACAGTATCGGACAGC & Amplify PcORP1 transcripts for quantitative real-time PCR \\
P14 & CAAACCCAGCAATGGAGTA & $\ldots$ \\
P15 & ACTGCACGTTCCAGACGATC & Amplify PcActin transcripts for quantitative real-time PCR \\
P16 & CCACCACCTTGATCTTCATG & $\ldots$ \\
P17 & TGCAATTCCCGCAGTCGT & Amplify PsORP1 transcripts for quantitative real-time PCR \\
P18 & GCGTACACCATCGCAGTCAGT & $\ldots$ \\
P19 & ACTGCACCTTCCAGACCATC & Amplify PsActin transcripts for quantitative real-time PCR \\
P20 & CCACCACCTTGATCTTCATG & $\ldots$ \\
\hline
\end{tabular}

${ }^{\mathrm{z}} \mathrm{PCR}=$ polymerase chain reaction. 
liquid $10 \% \mathrm{~V} 8$ medium. These plates were incubated in darkness at $25^{\circ} \mathrm{C}$ for 2 days. After washing the mycelia seven times in $30 \mathrm{ml}$ of sterile tap water, another $20 \mathrm{ml}$ of sterile water was added and the resulting preparation was incubated in darkness at $25^{\circ} \mathrm{C}$ for $24 \mathrm{~h}$ to induce sporangia production. For $P$. capsici, 10 hyphal tip plugs ( $5 \mathrm{~mm}$ in diameter) of each strain were placed into a Petri dish containing $20 \mathrm{ml}$ of sterile deionized water and incubated at $25^{\circ} \mathrm{C}$ under continuous light for $48 \mathrm{~h}$. The number of all sporangia formed on 30 plugs was counted.

To analyze zoospore production in $P$. sojae, all strains were cultured on $10 \% \mathrm{~V} 8$ agar medium for 10 days in darkness at $25^{\circ} \mathrm{C}$. Then, the plates containing mycelium were washed seven times using $30 \mathrm{ml}$ of sterile tap water for each washing. Finally, to induce sporangia production and zoospore release, another $15 \mathrm{ml}$ of sterile water was added, and the plates were incubated in darkness $25^{\circ} \mathrm{C}$ for 8 h. For P. capsici, all of the isolates were cultured on $10 \%$ V8 agar medium for 3 days in darkness at $25^{\circ} \mathrm{C}$. Next, all of the plates were incubated at room temperature under continuous light for another 5 days. Then, $20 \mathrm{ml}$ of sterile distilled water was added to the container and the plates were placed at $4^{\circ} \mathrm{C}$ for $30 \mathrm{~min}$, followed by room temperature for $30 \mathrm{~min}$. Finally, the zoospore suspension from one Petri dish was poured into a sterile beaker and the zoospores were counted using a hemocytometer.

Cyst germination was assessed by plating approximately 500 zoospores on $10 \% \mathrm{~V} 8$ agar medium. After $4 \mathrm{~h}$ of incubation in the dark at $25^{\circ} \mathrm{C}$, the percent germination of each isolate was observed by counting 100 cysts. Cysts were considered to have germinated if the length of their germ tube was greater than the diameter of the cyst. Each experiment contained three replica plates and all experiments were repeated three times.

To evaluate the pathogenicity of $P$. sojae, the first true leaves of 9- to 10-day-old soybean seedlings, cultivar Williams (rps), were used. Leaves were placed in Petri dishes containing wet tissue to prevent dehydration, and a mycelial plug was placed in the middle of each soybean leaf. For the pathogenicity assay of $P$. capsici, detached leaves of Nicotiana benthamiana were used. For each isolate, there were six replicate leaves. After inoculation, leaves were incubated at room temperature for 4 days before measuring the lesion area using Image $\mathbf{J}$ 1.x (Schneider et al. 2012). The experiment was repeated three times.
Quantitative real time polymerase chain reaction analysis of transcript levels. Quantitative real-time polymerase chain reaction (PCR) analysis of transcript levels was performed using the ABI7500fast sequence detection system (Applied Biosystems) and the Fast SYBR Green Master Mix (Thermo Fisher Scientific) with the primers listed in Table 1 and the protocol of the manufacturer. The relative quantities of the PCR products were calculated using the $2^{-\Delta \Delta \mathrm{Ct}}$ method and the PCACTIN and PSACTIN genes were used as a reference (Yan and Liou 2006) to normalize the quantification of $P c O R P 1$ and $P S O R P 1$ transcript levels, respectively. The entire experiment was conducted twice, and each experiment included three replicates for each treatment.

\section{RESULTS}

CRISPR/Cas9 system for the point mutation in P . capsici and validation of transformants. A total of eight colonies representing at least seven independent transformants showed oxathiapiprolin resistance (Figs. 2 and 3). T41-1 and T41-16 were obtained from the same transformation reaction and might not represent independent transformation events. The remaining six transformants were all obtained independently from one another. Sequencing results showed that T65-1 and T65-2 were both heterozygous at nucleotide 2,379. One allele was the wild type and the other allele contained the point mutation $\mathrm{G} \rightarrow \mathrm{T}$ at this position. The synonymous point mutations at the sg2180 target sequence introduced from pBS-HDR2 were also heterozygous (Fig. 2). Similarly, T41-1, T41-16, and T42-10 were heterozygous at nucleotide 2,173 ( $\mathrm{G}$ and $\mathrm{T}$ ). However, they were all homozygous for the synonymous point mutations at the sg2381 target sequence. Interestingly, in T47-1, T47-17, and T47-19, three nucleotides $(2,379,2,380$, and 2,381) were deleted at the Cas9 cleavage site and no other mutations were found. This 3-bp deletion resulted in a deletion of one amino acid (N837) (Fig. 2). This mutation was presumably produced by nonhomologous end joining (NHEJ), triggered by CRISPR/Cas9.

Phenotype of $\boldsymbol{P}$. capsici transformants. All eight transformants grew on V8 plates containing various concentrations of oxathiapiprolin, up to $0.5 \mu \mathrm{g} / \mathrm{ml}$ (Fig. 3A), whereas the wild-type

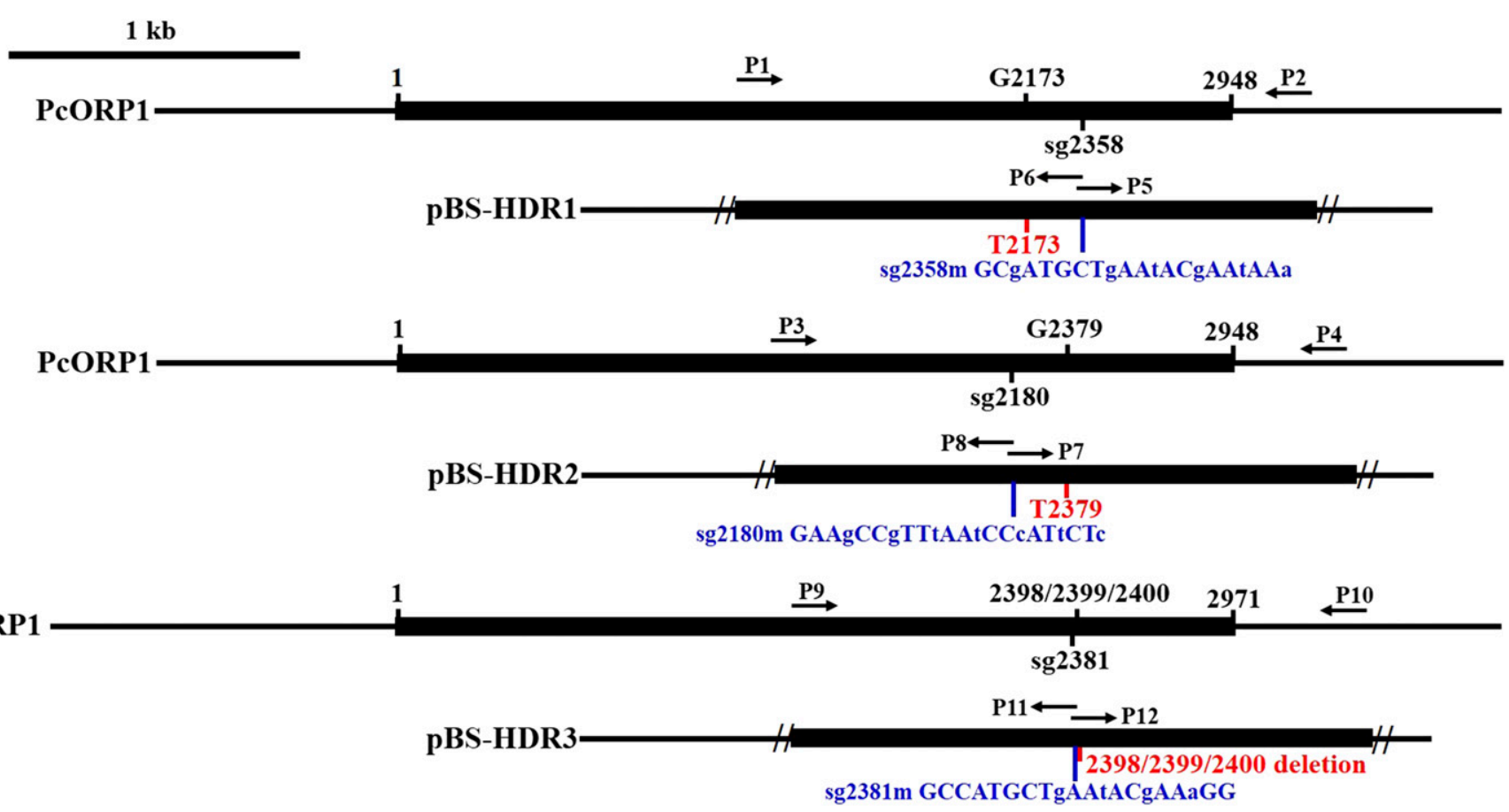

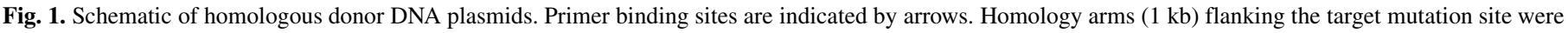
used. The subgenomic RNA recognition sites in pBS-HDR1, pBS-HDR2, and pBS-HDR3 were mutated and the new sequence was shown in red text. 
isolate BYA5 could not grow on V8 plates containing oxathiapiprolin at $0.005 \mu \mathrm{g} / \mathrm{ml}$. This confirms that individual heterozygous mutations of G770V or G839W in PcORP1 could produce high oxathiapiprolin resistance. The sensitivity results for T47-1, T4717 , and T47-19 suggested that the homozygous $\Delta \mathrm{N} 837$ mutation in PcORP1 also could confer high oxathiapiprolin resistance (Fig. 3A).

Surprisingly, neither T65-1 nor T65-2, containing the heterozygous point mutation G839W in PcORP1, could produce sporangia or zoospores, although they maintained pathogenicity (Table 2). By

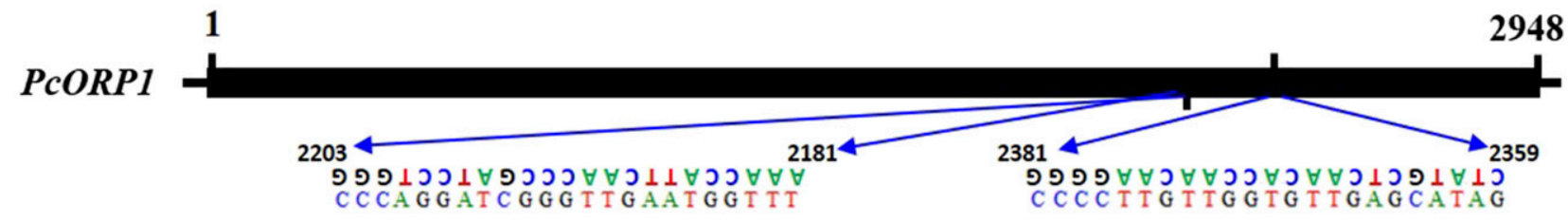

BYA5

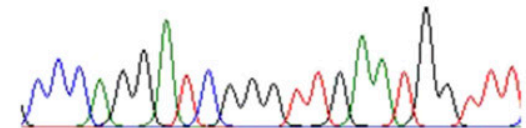

P. capsici

P. capsici

T41-1

T41-16

T42-10

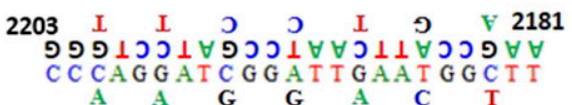

T65-1

T65-2

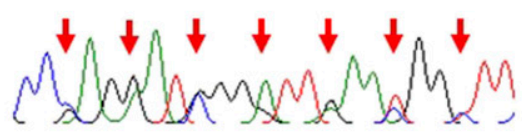

1

BYA5
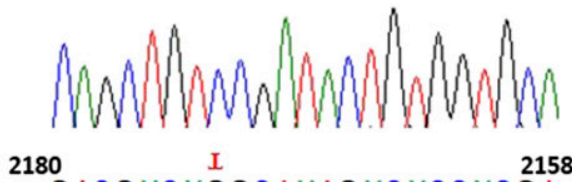

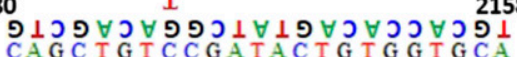

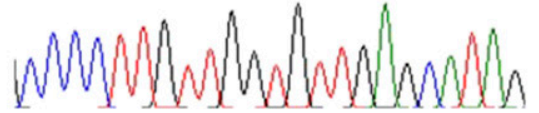

2381
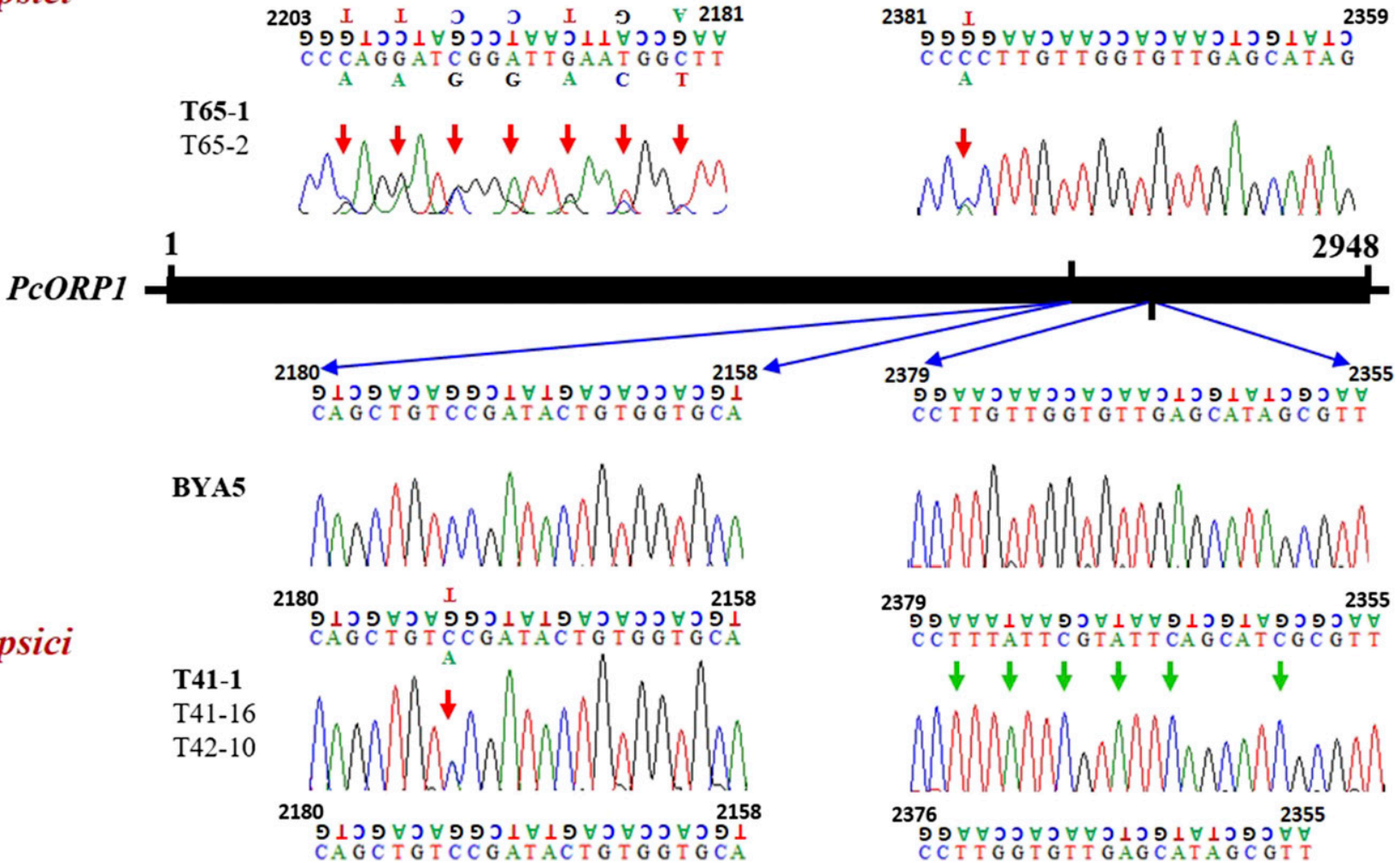

2379

פ $\forall \forall \forall \forall \forall \forall$ 年

C CT T TAT T C GTATICAGCATCGCGT

$\downarrow \downarrow \downarrow \downarrow \downarrow \downarrow$

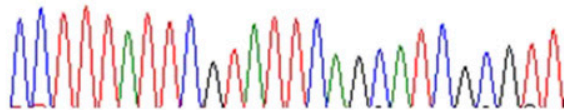

2376

פ

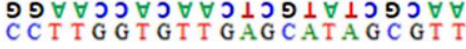

T47-1

T47-17

T47-19
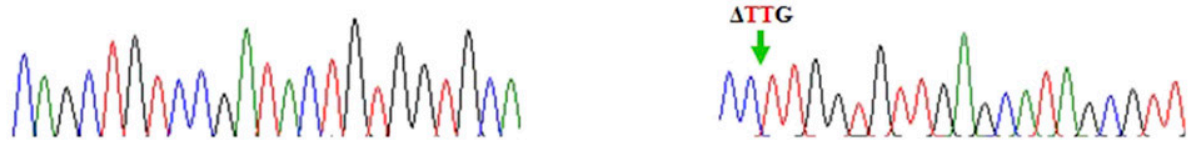

1

\section{PsORP1}
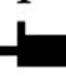

2402

כ $\forall \forall \forall ว \forall \forall \forall ว \forall כ \forall \forall ว \perp \supset \supset \perp \forall$

C CT T G T T G T G T GAG CAT

\section{P. sojae}

P6497

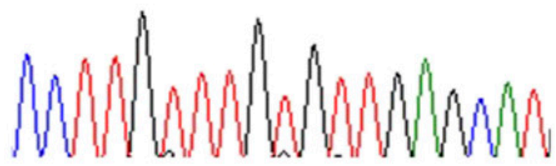

T4-71

T4-78

T4-51

$\mathrm{T} 4-52$

\section{9}

.


contrast, sporangia production, zoospore production, cyst germination, and pathogenicity in T41-1, T41-16 and T42-10 that contain the heterozygous mutation G770V were similar to that of the parental isolate BYA5, except for T41-1, which produced more sporangia than BYA5 (Table 2). T47-1, T47-17, and T47-19, carrying the homozygous mutation $\triangle \mathrm{N} 837$, showed normal cyst germination but somewhat lower sporangia and zoospore production and pathogenicity (Table 2) relative to the wild-type parent.

Phenotypes conferred by the $\mathbf{N 8 3 7}$ deletion in $P$. sojae. Four independent oxathiapiprolin-resistant colonies (T4-71, T4-78, T4-51, and T4-52) with the $\Delta$ N837 in PcORP1 were obtained using the CRISPR/Cas9 system and PYF515-sg2381 (sg2381: GCCA TGCTCAACACAAACAA) and pBS-HDR3 (Fig. 1) in P. sojae P6497. Interestingly, all four colonies were homozygous for $\triangle N 837$, even though the synonymous mutations in the sgRNA target sequence were heterozygous (Fig. 2). One $\triangle \mathrm{N} 837$ mutation might have been produced by NHEJ and one by HDR. All four strains showed high oxathiapiprolin resistance (Fig. 3B): they grew very well on V8 plates containing oxathiapiprolin concentrations up to $0.5 \mu \mathrm{g} / \mathrm{ml}$ (Fig. 3B), when the wild-type isolate P6497 could not grow on V8 plates containing oxathiapiprolin at $0.005 \mu \mathrm{g} / \mathrm{ml}$.

Compared with the wild-type P6497, all four P. sojae transformants containing the $\triangle \mathrm{N} 837$ mutation in PsORP1 showed normal cyst germination and a somewhat lower mycelial growth rate.
Sporangia and zoospore production were reduced slightly in T471 but were dramatically reduced in T4-78, T4-51, and T4-52. All four strains showed greatly reduced pathogenicity on soybean (Table 3).

Gene expression level analysis. Expression of $P c O R P l$ was lower in all of the P. capsici transformants than in BYA5 (Fig. 4A). In addition, the expression of $P C O R P 1$ differed between these eight transformants. Expression was nearly normal in T65-2 and T47-16 but very low in T65-1 and T47-1. Among the P. sojae transformants, expression of PSORP1 in T4-71 and T4-51 did not differ from the wild-type P6497. However, the expression of PsORPl appeared higher in T4-78 and lower in T4-52 (Fig. 4B).

\section{DISCUSSION}

Oxathiapiprolin has been used in China for approximately 2 years, and no resistant isolates of any oomycete pathogens have yet been reported in the field. However, resistant mutants could readily be obtained in the lab, and mutations G770V and G839W in the target in PcORP1 have been found in P. capsici oxathiapiprolinresistant mutants (Miao et al. 2016). Therefore, validating the genetic basis for resistance can provide the ground knowledge needed for rapid resistance monitoring and establishment of a resistance management strategy.
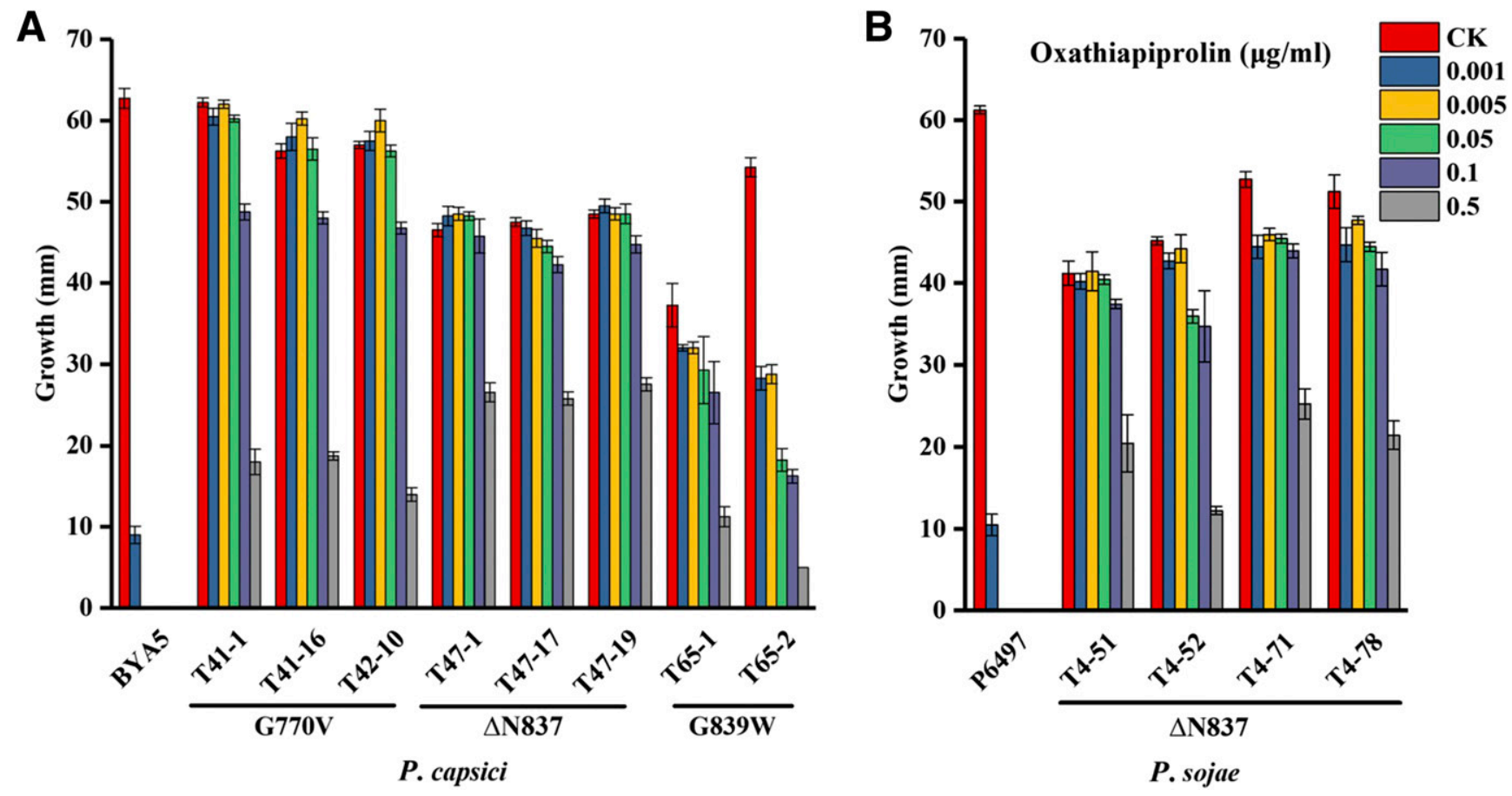

Fig. 3. Oxathiapiprolin sensitivity of CRISPR/Cas9 transformants (CK = control) for A, Phytophthora capsici and $\mathbf{B}$, P. sojae.

TABLE 2. Characteristics of Phytophthora capsici transformants and their parental isolates ${ }^{\mathrm{y}}$

\begin{tabular}{|c|c|c|c|c|c|c|}
\hline Isolate & Gene type & Mycelia growth (mm) & Sporangia $(n / \text { field of view })^{\mathrm{z}}$ & Zoospores $\left(10^{4} / \mathrm{ml}\right)$ & Cyst germination (\%) & Lesion area $\left(\mathrm{cm}^{2}\right)$ \\
\hline BYA5 & Wild type & $68 \pm 2 a$ & $148 \pm 42 \mathrm{bcd}$ & $97 \pm 21 \mathrm{ab}$ & $89 \pm 4 a$ & $3.4 \pm 0.5 \mathrm{ab}$ \\
\hline T65-1 & Heterozygous G839W & $41 \pm 4 \mathrm{f}$ & $0 \pm 0 \mathrm{f}$ & $0 \pm 0 \mathrm{e}$ & $\ldots$ & $2.1 \pm 1.4 \mathrm{abc}$ \\
\hline T65-2 & Heterozygous G839W & $59 \pm 2 \mathrm{~d}$ & $0 \pm 0 \mathrm{f}$ & $0 \pm 0 \mathrm{e}$ & $\ldots$ & $2.6 \pm 0.4 \mathrm{abc}$ \\
\hline $\mathrm{T} 41-1$ & Heterozygous G770V & $65 \pm 2 a b$ & $193 \pm 28 \mathrm{a}$ & $101 \pm 18 \mathrm{ab}$ & $90 \pm 2 \mathrm{a}$ & $3.9 \pm 0.1 \mathrm{a}$ \\
\hline T41-16 & Heterozygous G770V & $61 \pm 2 \mathrm{~cd}$ & $150 \pm 30 \mathrm{bc}$ & $90 \pm 14 \mathrm{bc}$ & $89 \pm 6 a$ & $2.4 \pm 1.8 \mathrm{abc}$ \\
\hline $\mathrm{T} 42-10$ & Heterozygous G770V & $63 \pm 2 b c$ & $163 \pm 40 \mathrm{ab}$ & $105 \pm 9 \mathrm{ab}$ & $85 \pm 3 a$ & $2.8 \pm 0.7 \mathrm{abc}$ \\
\hline $\mathrm{T} 47-1$ & Homozygous $\Delta \mathrm{N} 837$ & $51 \pm 1 \mathrm{e}$ & $113 \pm 16 \mathrm{de}$ & $75 \pm 7 \mathrm{~cd}$ & $90 \pm 3 a$ & $1.8 \pm 0.9 \mathrm{bc}$ \\
\hline $\mathrm{T} 47-17$ & Homozygous $\Delta \mathrm{N} 837$ & $53 \pm 1 \mathrm{e}$ & $106 \pm 13 \mathrm{e}$ & $69 \pm 9 d$ & $86 \pm 4 a$ & $1.2 \pm 0.7 \mathrm{c}$ \\
\hline T47-19 & Homozygous $\Delta \mathrm{N} 837$ & $53 \pm 2 \mathrm{e}$ & $126 \pm 13$ cde & $111 \pm 3 \mathrm{a}$ & $84 \pm 8 a$ & $1.6 \pm 1.9 \mathrm{bc}$ \\
\hline
\end{tabular}

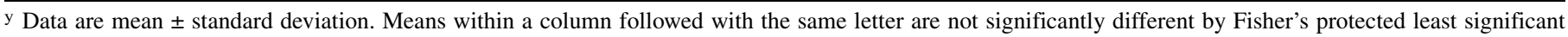
difference test $(P<0.05)$.

z Sporangia were counted using light microscope (magnification $\times 40)$. The number $(n)$ was the mean of 30 fields of view. 
Here, we successfully used the CRISPR/Cas9 system to obtain transformants heterozygous for the PcORP1 point mutation G839W and transformants heterozygous for the PcORP1point mutation G770V. This technology avoids the potentially undesirable effects of overexpression of target genes, frequent in the more traditional transformation methods. In addition, the point mutation G839W in PcORP1 was verified using traditional ectopic overexpression transformation. All five transformants showed high oxathiapiprolin resistance, confirming that G770V or G839W in PcORP1 do confer oxathiapiprolin resistance in P. capsici.

Because he CRISPR/Cas9 system can directly target a gene of interest for editing (Hsu et al. 2014), it usually generates transformants heterozygous at the edited locus. It is interesting that both the adaptation mutants and the CRISPR/Cas9 transformants were indeed heterozygotes for the G839W and G770V point mutations.
For $P$. capsici transformants T65-1 and T65-2, one allele was fully wild type and the other one was replaced via HDR, as expected. For T41-1, T41-16, and T42-10, the two alleles actually were replaced via HDR but the nonsynonymous point mutation was heterozygous. This might mean that only part of the donor template was used during HDR, because the distance from the sg2180 site and T2379 is approximately $200 \mathrm{bp}$. Paquet et al. (2016) also reported that homozygous mutations introduced using CRISPR/Cas9 require a guide RNA targeting close to the intended mutation. Therefore, when the CRISPR/Cas9 system is used to directly introduce a point mutation, the interval between the targeted mutation point and the guide RNA should also be considered during design of the constructs, especially for gene editing of recessive alleles such as CESA3 in oomycetes (Gisi et al. 2007). In T4-71, T4-78, T4-51, and T4-52 (which were all independent $P$. sojae transformants), the 3-bp

TABLE 3. Characteristics of Phytophthora sojae transformants and their parental isolates ${ }^{\mathrm{y}}$

\begin{tabular}{|c|c|c|c|c|c|c|}
\hline Isolate & Gene type & Mycelia growth (mm) & Sporangia $(n / \text { field of view })^{\mathrm{z}}$ & Zoospores $\left(10^{4} / \mathrm{ml}\right)$ & Cyst germination (\%) & Lesion area $\left(\mathrm{cm}^{2}\right)$ \\
\hline P6497 & Wild type & $66 \pm 1 \mathrm{a}$ & $26 \pm 3 a$ & $7.3 \pm 1.7 \mathrm{a}$ & $97 \pm 2 \mathrm{a}$ & $6.5 \pm 2.5 \mathrm{a}$ \\
\hline T4-71 & Homozygous $\Delta \mathrm{N} 837$ & $57 \pm 3 b$ & $17 \pm 6 b$ & $5.8 \pm 5.8 \mathrm{a}$ & $94 \pm 1 \mathrm{a}$ & $0.15 \pm 0.14 b$ \\
\hline T4-78 & Homozygous $\Delta \mathrm{N} 837$ & $56 \pm 2 b$ & $4.3 \pm 4 \mathrm{c}$ & $0.06 \pm 0.03 b$ & $95 \pm 3 a$ & $0.24 \pm 0.09 \mathrm{~b}$ \\
\hline $\mathrm{T} 4-51$ & Homozygous $\Delta \mathrm{N} 837$ & $46 \pm 2 d$ & $2.3 \pm 2 \mathrm{c}$ & $0.14 \pm 0.01 \mathrm{~b}$ & $96 \pm 2 a$ & $0.13 \pm 0.12 b$ \\
\hline $\mathrm{T} 4-52$ & Homozygous $\Delta \mathrm{N} 837$ & $50 \pm 1 \mathrm{c}$ & $4.0 \pm 4 \mathrm{c}$ & $0.06 \pm 0.02 \mathrm{~b}$ & $94 \pm 4 \mathrm{a}$ & $0.13 \pm 0.02 b$ \\
\hline
\end{tabular}

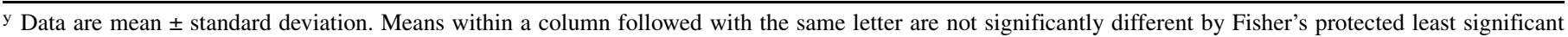
difference test $(P<0.05)$.

z Sporangia were counted using light microscope (magnification $\times 40)$. The number $(n)$ was the mean of 30 fields of view.
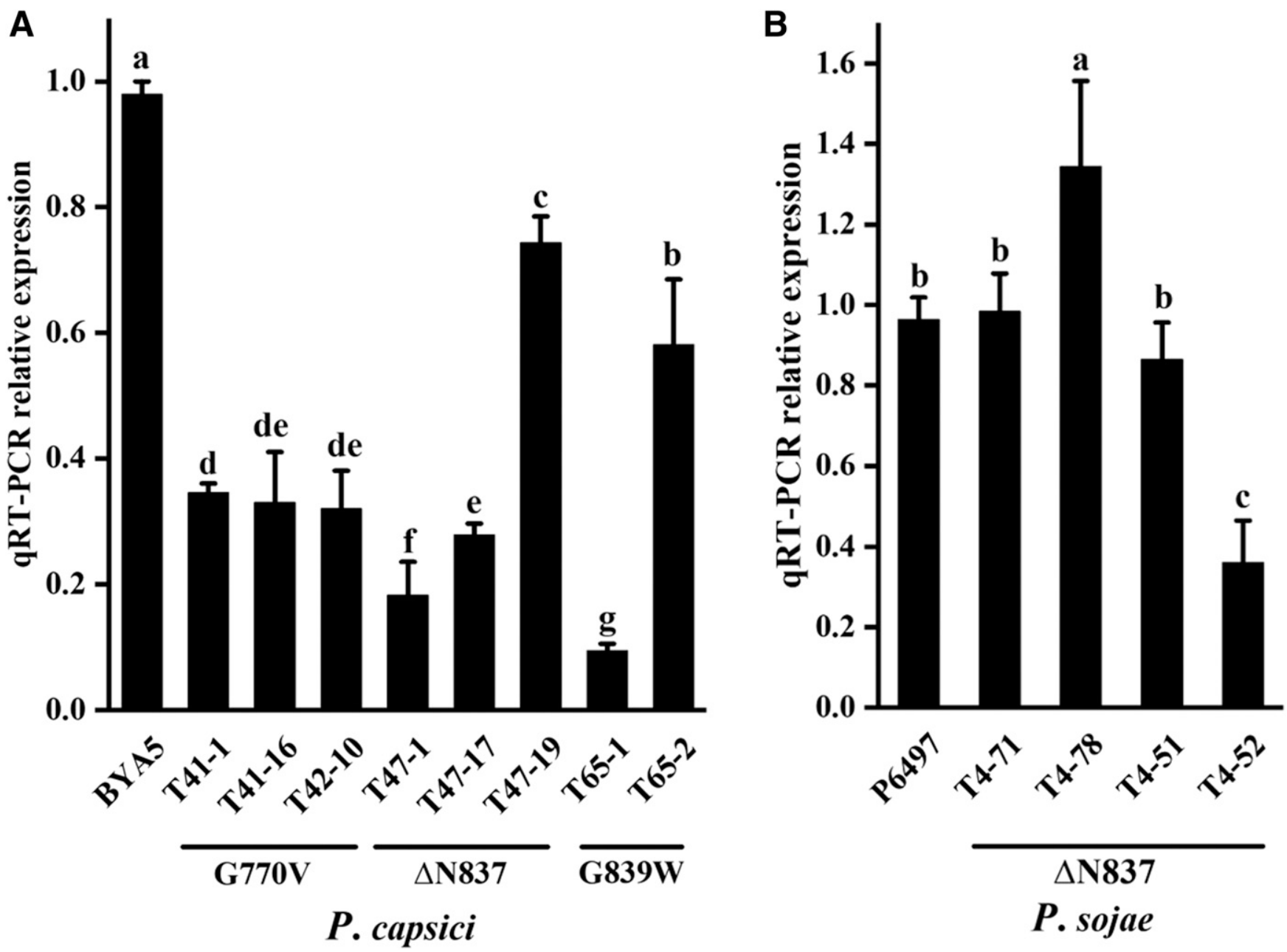

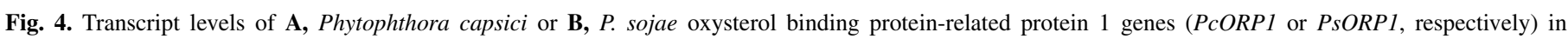

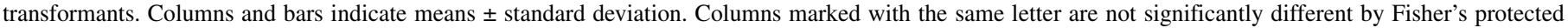
least significant difference test $(P<0.05)$. 
deletion was homozygous but the sg2381 target sequence emerged as heterozygous. We speculate that, after the sg2381-guided Cas9 cut the genome, one allele was repaired via NHEJ and the other one was repaired via HDR. If so, this would mean that the $\triangle \mathrm{N} 837$ mutation was produced seven times independently by NHEJ in the $P$. capsici and $P$. sojae transformants. We speculate that the tandem duplication of the codon CAA adjacent to the Cas9 cleavage site predisposed one of those codons to be lost during NHEJ.

The observation that the CRISPR/Cas9 transformants at G839W and G770V were all heterozygous, even when the synonymous mutations in the sgRNA target sequence had become homozygous, suggests that G839W and G770V might have severe fitness costs, or may be lethal, when they are homozygous. In a previous study (Miao et al. 2016), heterozygous G839W mutants in PcORP1 displayed high levels of fitness, and they could produce similar numbers of sporangia and zoospores compared with their parental isolate LP3. However, T65-1 and T65-2 with G839W in PcORP1, obtained independently from the wild-type isolate BYA5, could not produce sporangia. We speculate that the CRISPR/Cas9 treatment, or the transformation itself, might have caused some off-target deleterious mutations in these two lines. In a controversial study, Schaefer et al. (2017) suggested that CRISPR-Cas9 could induce more than 1,000 unexpected mutations in mice. However, this result has been questioned (Wilson et al. 2017), and deep sequencing of CRISPR/Cas9-modified Arabidopsis genomes did not detect any off-target mutations (Feng et al. 2014). An alternative hypothesis is that the original G839W mutants of $P$. capsici may have benefited from polymorphisms in their parent strain LP3 that compensated for the negative effects of G839W on sporangiogenesis, or that compensating mutations may have arisen during the fungicide adaptation process. More independent transformants carrying G839W are needed to resolve whether and, if yes, how this mutation truly interferes with sporangiogenesis.

Preliminary studies also had indicated that six independent $P$. capsici heterozygous mutants carrying G770V in PcORP1 had lower fitness and produced much less sporangia compared with their parental isolate HNJZ10. However, here, transformants T41-1, T41-16, and T42-10, all heterozygous at the G770V locus, showed similar fitness compared with parental isolate BYA5. The different effects of G770V in the two studies could possibly be related to differences in the genetic backgrounds of the parent isolates, HNJZ10 and BYA5. Nonetheless, our results indicate that the point mutation G770V in PcORP1 also has survival potential in the field. In future, this type of mutation should also be monitored.

Our results have shown for the first time that the mutation $\triangle \mathrm{N} 837$ in PcORP1 or PsORP1 could confer oxathiapiprolin resistance in P. capsici or P. sojae, respectively. Andreassi et al. (2013) previously observed that amino-acid substitutions (I, F, or Y) at N837 could confer oxathiapiprolin resistance in $P$. capsici but deletions were not previously observed. Amino-acid substitutions are the most common resistance mechanism against fungicides with a single site of action (Ma and Michailides 2005). However, amino-acid deletions in target proteins also can cause fungicide resistance in plant pathogens by altering the molecular target of the active ingredient. For example, $\Delta \mathrm{Y} 459 / \mathrm{G} 460$ was found in Cyp5 1 from Mycosphaerella graminicola field isolates resistant to sterol synthesis inhibitors (Leroux and Walker 2011), and the mutation $\Delta \mathrm{L} 497$ in the transcription factor mrr1 was detected in all Botrytis cinerea multidrug-resistant isolates analyzed by Leroch et al. (2012). Bittner et al. (2017) also detected the deletion $\Delta$ G818/F819 in PnORP1 in P. nicotianae oxathiapiprolin-resistant mutants produced by UV mutagenesis, although that mutation was not verified to cause resistance. We speculate that $\Delta \mathrm{N} 837$ in PcORP1 or PsORP1 causes a structural change in the ORD domain of these two proteins. This change may reduce the binding affinity of oxathiapiprolin to its target protein. The fitness results for $P$. capsici CRISPR/Cas9 transformants T47-1, T47-17, and T47-19 and P. sojae CRISPR/Cas9 transformants T4-71, T4-78, T4-51, and T4-52 suggested that the mutants with $\Delta \mathrm{N} 837$ may have survival potential in the field. Therefore, the $\Delta \mathrm{N} 837$ oxathiapiprolin resistance mutation should also be monitored in the future.

In conclusion, our results using CRISPR/Cas9 confirmed that the point mutations G770V and G839W in PcORP1 do confer resistance to oxathiapiprolin in $P$. capsici. Notably, we also found a new point mutation, $\triangle \mathrm{N} 837$, in PcORP1 that could produce oxathiapiprolin resistance in $P$. capsici and $P$. sojae. Mutants with any of these three point mutations may have survival potential in the field. This information will increase our understanding of oxathiapiprolin resistance in plant-pathogenic oomycetes and should be useful for the development of new fungicides.

\section{ACKNOWLEDGMENTS}

We thank DuPont Crop Protection for providing oxathiapiprolin, F. Arredondo (Oregon State University, Corvallis) for useful advice, members of the Tyler Laboratory for experimental assistance, and the Center for Genome Research and Biocomputing at Oregon State University for DNA sequencing.

\section{LITERATURE CITED}

Andreassi, J. L., II, Gutteridge, S., Pember, S. O., and Sweigard, J. A. 2013. Detection and screening method and materials useful in performance thereof. International Patent No. WO2013009971. World Intellectual Property Organization, Geneva.

Bittner, R. J., and Mila, A. L. 2017. Efficacy and timing of application of oxathiapiprolin against black shank of flue-cured tobacco. Crop Prot. 93: 9-18.

Bittner, R. J., Sweigard, J. A., and Mila, A. L. 2017. Assessing the resistance potential of Phytophthora nicotianae, the causal agent of black shank of tobacco, to oxathiopropalin with laboratory mutants. Crop Prot. 102:63-71.

Blair, J. E., Coffey, M. D., Park, S. Y., Geiser, D. M., and Kang, S. 2008. A multi-locus phylogeny for Phytophthora utilizing markers derived from complete genome sequences. Fungal Genet. Biol. 45:266-277.

Cohen, Y. 2015. The novel oomycide oxathiapiprolin inhibits all stages in the asexual life cycle of Pseudoperonospora cubensis-causal agent of cucurbit downy mildew. PLoS One 10:e0140015.

Derevnina, L., Petre, B., Kellner, R., Dagdas, Y. F., Sarowar, M. N., Giannakopoulou, A., Concepcion, J. C. D., Chaparro-Garcia, A., Pennington, H. G., van West, P., and Kamoun, S. 2016. Emerging oomycete threats to plants and animals. Philos. Trans. R. Soc. B. 371:20150459.

Doench, J. G., Hartenian, E., Graham, D. B., Tothova, Z., Hegde, M., Smith, I., Sullender, M., Ebert, B. L., Xavier, R. J., and Root, D. E. 2014. Rational design of highly active sgRNAs for CRISPR-Cas9-mediated gene inactivation. Nat. Biotechnol. 32:1262-1267.

Edwards, K., Johnstone, C., and Thompson, C. 1991. A simple and rapid method for the preparation of plant genomic DNA for PCR analysis. Nucleic Acids Res. 19:1349.

Fang, Y., Cui, L., Gu, B., Arredondo, F., and Tyler, B. M. 2017. Efficient genome editing in the oomycete Phytophthora sojae using CRISPR/Cas9. Curr. Protoc. Microbiol. 44:21A.1.1-21A.1.26.

Fang, Y., and Tyler, B. M. 2016. Efficient disruption and replacement of an effector gene in the oomycete Phytophthora sojae using CRISPR/Cas9. Mol. Plant Pathol. 17:127-139.

Feng, Z., Mao, Y., Xu, N., Zhang, B., Wei, P., Yang, D. L., Wang, Z., Zhang, Z., Zheng, R., Yang, L., Zeng, L., Liu, X., and Zhu, J. K. 2014. Multigeneration analysis reveals the inheritance, specificity, and patterns of CRISPR/Cas-induced gene modifications in Arabidopsis. Proc. Natl. Acad. Sci. USA 111:4632-4637.

Fichtner, E. J., Rizzo, D. M., Kirk, S. A., and Webber, J. F. 2012. Infectivity and sporulation potential of Phytophthora kernoviae to select North American native plants. Plant Pathol. 61:224-233.

FRAC. 2017. FRAC Code List 2017: Fungicides sorted by mode of action (including FRAC Code numbering). Online publication. Fungicide Resistance Action Committee (FRAC). http://www.frac.info/what-s-new/ 2017/03/03/publication-of-the-frac-code-list-2017

Gisi, U., and Sierotzki, H. 2008. Fungicide modes of action and resistance in downy mildews. Eur. J. Plant Pathol. 122:157-167.

Gisi, U., Waldner, M., Kraus, N., Dubuis, P. H., and Sierotzki, H. 2007. Inheritance of resistance to carboxylic acid amide (CAA) fungicides in Plasmopara viticola. Plant Pathol. 56:199-208.

Hsu, P. D., Lander, E. S., and Zhang, F. 2014. Development and applications of CRISPR-Cas9 for genome engineering. Cell 157:1262-1278. 
Ji, P., and Csinos, A. S. 2015. Effect of oxathiapiprolin on asexual life stages of Phytophthora capsici and disease development on vegetables. Ann. Appl. Biol. 166:229-235.

Ji, P., Csinos, A. S., Hickman, L. L., and Hargett, U. 2014. Efficacy and application methods of oxathiapiprolin for management of black shank on tobacco. Plant Dis. 98:1551-1554.

Leroch, M., Plesken, C., Weber, R. W., Kauff, F., Scalliet, G., and Hahn, M. 2012. Gray mold populations in German strawberry fields are resistant to multiple fungicides and dominated by a novel clade closely related to Botrytis cinerea. Appl. Environ. Microbiol. 79:159-167.

Leroux, P., and Walker, A. S. 2011. Multiple mechanisms account for resistance to sterol $14 \alpha$-demethylation inhibitors in field isolates of Mycosphaerella graminicola. Pest Manage. Sci. 67:44-59.

Ma, Z., and Michailides, T. J. 2005. Advances in understanding molecular mechanisms of fungicide resistance and molecular detection of resistant genotypes in phytopathogenic fungi. Crop Prot. 24:853-863

Mair, W., Lopez-Ruiz, F., Stammler, G., Clark, W., Burnett, F., Hollomon, D., Ishii, H., Thind, T. S., Browm, J. K. M., Fraaije, B., Cools, H., Shaw, M., Fillinger, S., Walker, A., Mellado, E., Schnabel, G., Mehl, A., and Oliver, R. P. 2016. Proposal for a unified nomenclature for target-site mutations associated with resistance to fungicides. Pest Manage. Sci. 72:1449-1459.

Meitz-Hopkins, J. C., Pretorius, M. C., Spies, C. F. J., Huisman, L., Botha, W. J., Langenhoven, S. D., and McLeod, A. 2014. Phytophthora species distribution in South African citrus production regions. Eur. J. Plant Pathol. 138:733-749.

Miao, J. Q., Cai, M., Dong, X., Liu, L., Lin, D., Zhang, C., Pang, Z. L., and Liu, X. L. 2016. Resistance assessment for oxathiapiprolin in Phytophthora capsici and the detection of a point mutation (G769W) in PcORP1 that confers resistance. Front. Microbiol. 7:615.

Pang, Z., Shao, J., Chen, L., Lu, X., Hu, J., Qin, Z., and Liu, X. 2013. Resistance to the novel fungicide pyrimorph in Phytophthora capsici: Risk assessment and detection of point mutations in CesA3 that confer resistance. PLoS One 8:e56513.

Paquet, D., Kwart, D., Chen, A., Sproul, A., Jacob, S., Teo, S., Olsen, K. M., Gregg, A., Noggle, S., and Tessier-Lavigne, M. 2016. Efficient introduction of specific homozygous and heterozygous mutations using CRISPR/Cas9. Nature 533:125-129.

Pasteris, R. J., Hanagan, M. A., Bisaha, J. J., Finkelstein, B. L., Hoffman, L. E., Gregory, V., Andreassi, J. L., Sweigard, J. A., Klyashchitsky, B. A., and Henry, Y. T. 2016. Discovery of oxathiapiprolin, a new oomycete fungicide that targets an oxysterol binding protein. Bioorg. Med. Chem. 24:354-361.

Schaefer, K. A., Wu, W. H., Colgan, D. F., Tsang, S. H., Bassuk, A. G., and Mahajan, V. B. 2017. Unexpected mutations after CRISPR-Cas9 editing in vivo. Nat. Methods 14:547-548.

Schneider, C. A., Rasband, W. S., and Eliceiri, K. W. 2012. NIH Image to ImageJ: 25 years of image analysis. Nat. Methods 9:671-675.

Urban, J., and Lebeda, A. 2006. Fungicide resistance in cucurbit downy mildew-methodological, biological and population aspects. Ann. Appl. Biol. 149:63-75.

Wilson, C. J., Fennell, T., Bothmer, A., Maeder, M. L., Reyon, D., Cotta-Ramusino, C., Fernandez, C. A., Marco, E., Barrera, L. A., Jayaram, H., Albright, C. F., Cox, G. F., Church, G. M., and Myer, V. E. 2017. The experimental design and data interpretation in 'Unexpected mutations after CRISPR Cas9 editing in vivo' by Schaefer et al. are insufficient to support the conclusions drawn by the authors. Online publication. bioRxiv.

Yan, H. Z., and Liou, R. F. 2006. Selection of internal control genes for realtime quantitative RT-PCR assays in the oomycete plant pathogen Phytophthora parasitica. Fungal Genet. Biol. 43:430-438.

Ziogas, B. N., Markoglou, A. N., Theodosiou, D. I., Anagnostou, A., and Boutopoulou, S. 2006. A high multi-drug resistance to chemically unrelated oomycete fungicides in Phytophthora infestans. Eur. J. Plant Pathol. 115: 283-292. 